\title{
Tiempo y memoria en el proceso de conocimiento de las ciencias de la información
}

Time and memory in the information science knowledge process

Héctor Guillermo Alfaro López

Universidad Nacional Autónoma de México. Instituto de

Investigaciones Bibliotecológicas, México

logos_y_cosmos@yahoo.com.mx

(iD https://orcid.org/0000-0003-2143-7247

\begin{abstract}
Resumen:
Tiempo y memoria son parte constitutiva de la información y, más aún, son su condición de posibilidad. Para comprender y fundamentar cognoscitivamente tal afirmación en las ciencias de la información, se lleva a cabo una reflexión dividida en dos fases: en la primera se busca responder qué son el tiempo y la memoria; en el caso del tiempo, a partir de la propuesta del sociólogo Norbert Elias que define el tiempo como "símbolo social comunicable" y, respecto a la memoria se hacen una serie de consideraciones a partir de su plasmación en las textualidades. En la segunda, fase se lleva a cabo la fundamentación epistemológica del tiempo y la memoria, para estatuirlos como conceptos que tienen la función de ser soporte y operativos de los otros conceptos en las ciencias de la información con que construyen sus objetos de conocimiento. Todo este proceso de conocimiento se ejemplifica en el caso de la lectura, dentro del marco de la bibliotecología; para ello se implementan los conceptos relacionales de intermediario, mediadortraducción, acuñados por Bruno Latour. Se muestra cómo la lectura transporta la información en cuanto significado, que puede ser conducido como mediador-traductor o intermediario, a cuya base se encuentran el tiempo y la memoria.
\end{abstract}

Palabras clave: Tiempo, Memoria, Conocimiento, Ciencias de la Información.

\section{ABstract:}

Time and memory are a constitutive part of information and, even more, are its condition of possibility. To understand and cognitively substantiate such a statement in the information sciences, a reflection is carried out divided into two phases: in the first, it seeks to answer what are time and memory. In the case of time, based on the proposal of the sociologist Norbert Elias who defines time as: "a communicable social symbol" and, with respect to memory, a series of considerations are made base on its expression in textualities. In the second phase, the epistemological foundation of time and memory is carried out, to establish them as concepts that have the function of being support and operative of the other concepts in the information sciences with which they build their objects of knowledge. All this process is exemplified in the case of reading, within the framework of librarianship; for this they are implemented in the relational concepts of intermediary, mediator-translation, created by Bruno Latour. Showing how reading carries information as meaning, which can be conducted as a mediator-translator or intermediary, at the base of which time and memory are found.

KeYwords: Time, Memory, Knowledge, Information Sciences.

\section{Tiempo}

La información nunca se encuentra estática ni se desvanece sin dejar rastros una vez que ha sido enunciada. Muy por el contrario, está perpetuamente en movimiento o, mejor aún, en un amplio espectro de movimientos, configuraciones y desplazamientos multidireccionales; y esto asimismo va acompañado de una amplia cobertura de dispositivos para su preservación. Tales atributos nos remiten a dos elementos sustanciales de la información: tiempo y memoria. Aunque no tuviéramos una concepción fundamentada de semejante declaración de manera intuitiva algo nos diría que a la base de la información y del amplio proceso informacional en su conjunto gravitan el tiempo y la memoria. Y, más aún, que sin tales factores 
la información no sería lo que es del todo. Con un poco que se afine esa intuición tendríamos una primera noción de la presencia y significación del tiempo y la memoria en el interior de la información: desde el momento en que la información se genera, sea en el ámbito que sea, podemos percibir su movimiento a través de sus desplazamientos en la sociedad al ser transmitida y recepcionada a través de los diversos agentes e instancias, con lo que asimismo observamos sus transfiguraciones y trayectorias a lo largo de un período. También en ese recorrido podemos observar los diversos esfuerzos sociales que se hacen por preservar esa información, para que no se pierda y pueda volver a recuperarse. Lo que redunda en que se mantenga viva tal información y siga desplazándose socialmente e incluso a través de las generaciones.

Si damos un paso más allá de la mera percepción de la movilidad inmediata de la información a través del tiempo y consideramos más reflexivamente esto, se puede adelantar el supuesto de que el tiempo no es algo exterior a la información y que se manifiesta a partir de sus desplazamientos a través de periodos de tiempo en la sociedad; sino que el tiempo y su correlato que es la memoria, son parte constitutiva de la información $y$, en cuanto tal, son también su condición de posibilidad. Lo que por otra parte nos ubica de lleno en un plano ya no sólo perceptivo sino plenamente cognoscitivo y con él las preguntas surgen al paso: ¿De qué clase de tiempo y memoria (y su relación) estamos hablando? ¿Cómo es que tiempo y memoria se constituyen en instancias del proceso de conocimiento de las ciencias de la información?

El tiempo es una noción que desde los orígenes de la conciencia reflexiva ha desvelado la razón de los seres humanos. Lo que ha producido una ingente cantidad de propuestas, concepciones e ideas de diversa índole para dar explicación de lo que es, de lo que significa, de lo que implica... las dos concepciones de mayor elaboración y que acabaron por decantarse para establecerse como las más precisas y autorizadas son aquellas de raigambre filosófica y de la física. El tema del tiempo de una u otra forma es una sombra que ha acompañado a la filosofía a lo largo de toda su historia. Pero es en la época moderna con el ascenso del individuo y su divinización que lo retraía hacia su propia interioridad (entendida de manera racionalista) y que lo aislaba a su vez dentro de la colectividad, que el tema del tiempo va a tomar un giro diferente gestándose en el trayecto que va de Descartes a Kant. Este último filósofo es el hace su enunciación más popular y definitiva, determinando de diversos modos las posteriores concepciones humanas y sociales del tiempo. Para Kant el tiempo, así como el espacio son, en su propia conceptografía, categorías a priori del entendimiento; en otras palabras, el tiempo es una forma innata de la experiencia humana, la cual es proyectada hacia el exterior otorgándole temporalidad a la realidad. De esta manera el tiempo es concebido como una entidad subjetiva. Por el lado de la física, se considera que el tiempo es un factor objetivo del espacio natural: es igual que el resto de los objetos naturales con la única diferencia de no ser perceptible. De manera análoga a lo que significó Kant, el gran formulador de esta concepción en la física fue Isaac Newton, él consolida el estatus del tiempo como objetividad. Aunque con Albert Einstein la visión del tiempo dentro de la física va a sufrir profundos cambios. Tanto la postura subjetiva como la objetiva coinciden en considerar al tiempo como un hecho natural existente, en un caso como "algo que existe" como una representación subjetiva de los humanos, en el otro caso "existe por sí mismo" independiente del hombre. Pero además hay otro factor subrepticio en el que coinciden ambas posturas opuestas: tanto el enfoque subjetivo como el objetivo son concebidos y formulados desde la perspectiva del sujeto individual, sea este designado como el filósofo o el físico o el individuo de a pie; con lo que se soslaya al conjunto social y con él su cauda histórica. Si estos aspectos pasados por alto se toman en consideración y se les da un fundamento interpretativo pueden ofrecer una tercera vía como opción ante la disyuntiva entre las concepciones subjetiva y objetiva del tiempo y, en cuanto tal, una base de sustentación de conocimiento de las ciencias de la información. Para ello es pertinente que acudamos a las reflexiones que sobre el tiempo emprendió el gran sociólogo Norbert Elías, el cual en sus propias palabras nos dicen que entiende por tiempo:

Así pues, con la palabra «tiempo» nos remitimos a la puesta en relación de posiciones y periodos de dos o más procesos factuales, que se mueven continuamente. Los acontecimientos en curso son perceptibles, pero la relación entre ellos constituye una elaboración de percepciones que hacen hombres con ciertos conocimientos. Queda expresada en un símbolo 
social comunicable: el concepto tiempo que en una sociedad determinada y con la ayuda de un modelo sensible formado por sonidos, puede comunicar de un hombre a otro de imagen mnemotécnica, experimentable, aunque no perceptible a los sentidos.

Siguiendo la costumbre de la antigua epistemología, sugeriría aquí la cuestión de si el tiempo es sólo una relación que hace el hombre, y no algo que exista de una manera real e independiente de él. Falso planteamiento cuyo defecto residente otras cosas, partir de un individuo como sujeto del conocimiento. Pues bien, el individuo no inventa el concepto de tiempo por sí mismo, sino que aprende desde su infancia tanto el concepto de tiempo como la institución social del tiempo que le está unida de modo indisoluble, ir creciendo en una sociedad a la cual pertenecen ese concepto y esa institución. En ella, el individuo aprende el concepto del tiempo no sólo como medio de la reflexión que verá la luz en libros filosóficos" (Elías, 1989, pp. 20-21).

Como queda de manifiesto en las palabras de Elías, el tiempo es concebido desde la perspectiva claramente sociológica, pero llevando agua al molino de las ciencias de la información puede ser interpretado y asumido de la siguiente forma: primero, es de remarcar los puntos centrales de semejante concepción; subrepticiamente queda de manifiesto que, a diferencia de las ya explicadas postura subjetiva y objetiva, para Elías el tiempo no es un "hecho natural existente", puesto que para él es un simbolo social comunicable, caracterización de suma importancia, en cuanto tal ese símbolo es comunicado de un individuo a otro; comunicación mediada por la memoria (sobre la cual se hará una más detallada explicación adelante), lo que es comunicado es la percepción de las relaciones de los acontecimientos en que se encuentran sumergidos los individuos. Lo que les brinda un marco de referencia para orientarse socialmente a través de la obtención de conocimientos. El colofón de esta concepción es que tal gestación y asunción del tiempo no es producto de un individuo aislado y ahistórico, sino todo lo contrario, es el resultado de un largo proceso civilizatorio de la humanidad en su conjunto y que a través de una constante individualización es incorporado en la conciencia de cada persona. Y así es también comunicado el símbolo de tiempo entre individuos.

Con que agudicemos la mirada podemos vislumbrar en esta breve síntesis de los elementos centrales de la concepción del tiempo de Elías que la cuestión de la información asoma en cada intersticio: el símbolo de tiempo que es comunicado entre los individuos conlleva información, el cual es producto de las percepciones que se captan de las relaciones entre los acontecimientos. Cada acontecimiento, por su propia naturaleza y su dinámica, genera contenidos informativos. Información que se constituye conocimiento, que a su vez se establece como el marco de referencia para orientarse socialmente los individuos. Y todo esto es el resultado de un proceso de longue dureé civilizatorio, qué va acumulando información a través de su despliegue histórico para desembocar en la conciencia individualizada de cada individuo. Es de acotar la importancia del lenguaje o, más aún, de los distintos lenguajes en la transmisión de la información; cada lenguaje tiene el atributo de articularse a partir de signos y símbolos y es en función de estos que comunica la información. Y entre tales símbolos, el del tiempo tiene un gran poder de aglutinar y transmitir información y con ello constituir sólidos enlaces de organización y cohesión social. Comprendido todo esto desde la perspectiva de la propia información implica que está determinada por movimientos y desplazamientos permanentes signados por la temporalización. Por lo que la información, siguiendo los supuestos de Elías, tiene como condición de posibilidad de su generación y desenvolvimiento social el tiempo.

\section{Memoria}

El texto, dispositivo de la memoria par excellence, es también objeto que vehiculiza a través del tiempo la información en él contenida y a su vez esa información es tiempo. Para clarificar qué debemos entender por memoria el texto nos puede dar las claves para ello. Antes de la aparición del texto y con él la palabra escrita, la comunicación y lo que con ella se informaba se realizaba por vía de la oralidad, lo que significa ubicarse históricamente en aquellas sociedades sustentadas en la cultura oral. La sociedad en que está bastante documentada la transición de la cultura oral a la cultura escrita, es la griega antigua. En esta sociedad el tránsito de una forma cultural a otra significó con ello la reconstitución de la memoria. La Ilíada, el 
poema fundamental de la cultura griega, nace en una sociedad oral, lo que significa que era transmitido oralmente por aedos o por el propio pueblo, para lo cual era conservado en una memoria colectiva, la cual actuaba transfigurando imaginativa y creativamente el poema, porque lo que sobre un núcleo temático básico, la guerra de Troya, se podía reconstituir agregándole pasajes nuevos al ser comunicado de unos a otros individuos. Era una información moviente, no estabilizada en su preservación en la memoria colectiva, que al pasar por la individualización seguía reconstituyéndose una y otra vez en la memoria de cada individuo. Al ingresar la escritura en el mundo griego la Ilíada fue gradualmente estabilizada en una textualidad. Lo que significó descargar la memoria colectiva e individual del recuerdo del poema. Así la palabra escrita estandarizada dentro de la textualidad pasó a constituirse en otra forma de memoria con sus normas propias, diferenciadas de la memoria colectiva e individual. Por su parte, la memoria individual quedó restringida a preservar en la memoria aquello que atañe privilegiadamente a la existencia propia de la persona. Detengámonos en este punto nodal en la explicación sobre la memoria.

El texto, cuyo soporte canónico y fetichizado es el libro, en cualquiera de sus materialidades una vez que ha sido fijado se convierte en sí mismo en memoria de la información en él contenida, lo que le pone en disposición de trasportarse de una sociedad a otra y trasladarse, incluso, a través de las centurias para llegar a las manos de un lector. El libro, que en sí es ya a un dispositivo de preservación de información puede pasar por otros dispositivos de conservación de información como pueden ser, por ejemplo, las bibliotecas. Ahora bien, todos estos dispositivos tienen un denominador común en cuánto memoria fija, estabilizada: evitar mayormente la pérdida de información: la prioridad es su conservación para su transmisión. Por su parte, la memoria individual de alguna manera conserva las cualidades de la memoria propia de la cultura oral, recrea su información. El recuerdo del pasado se encuentra mediado por el olvido. El mecanismo para filtrar y deshacerse de información es el olvido, que responde normalmente a directrices existenciales. Pero el recuerdo pasado por la criba del olvido reconfigura la información preservada en la memoria: modifica o crea nueva información.

La información fija que proporcionan los dispositivos de preservación es aquella que se genera y proyecta a través del largo proceso civilizatorio, la cual al ser transmitida (lo que simbólicamente es una especie de recuerdo, que pretende evadir el olvido) propicia en sus receptores todo un entramado reconstituyente de la información. Este tipo de información que se desplaza a través de los siglos es el basamento mnemotécnico sobre el que se erige la memoria como acto de representación que permiten desplegar la facultad humana de síntesis para la determinación del tiempo, cómo le explica también Elías:

\footnotetext{
Clave para resolver las cuestiones del tiempo y de la determinación del tiempo es, en realidad, de facultad específica humana para tener una vista de conjunto y relacionar lo que, es una serie continua de hechos, sucede «más temprano»o «más tarde», «antes» o «después». Papel fundamental desempeña la memoria en este acto de representación en que vemos junto lo que no sucedió al mismo tiempo. Así pues, si me refiero a la facultad de sintetizar, hablo en particular de la capacidad humana de imaginar cómo presente algo que, realidad, no lo está, y relacionarlo con lo que, en verdad, sucede aquí y ahora. Se trata sólo de una manifestación de la facultad humana de síntesis, qué desempeña un papel decisivo en todas las formas de determinar el tiempo. (...) Es realidad, este concepto representa un nexo instrumental nada sencillo de sucesos que grupos humanos más o menos organizados establecen para ciertos fines, entre y dentro de continuos en devenir observables, se incluyan o no a sí mismos, como el continuum que constituyen (Elías, 1989, p. 86).
}

De hecho con lo que explican estas palabras, tenemos la intersección de dos órdenes de la memoria, con sus respectivos y específicos contenidos informacionales: la memoria histórico-social la cual se individualiza en la memoria personal; con lo que además se da la conjunción de tiempo y memoria recorridas por el flujo de información. Así el símbolo social de tiempo puede ser constituido y comunicado entre los individuos. Una vez que se ha dado respuesta a la cuestión de lo que aquí se entiende como tiempo y memoria, así como la relación que guardan, queda asimismo de manifiesto que tal explicación ha tenido como marco de referencia el mundo social, de la vida cotidiana. Lo que ha permitido seguir y comprender escorzos de los movimientos y 
desplazamientos de la información signadas por el tiempo y la memoria, por lo que ahora se requiere realizar el tránsito hacia el ámbito del conocimiento sistemático de la ciencia.

Es claro que el mundo de la vida cotidiana y el ámbito de la ciencia son realidades profundamente diferenciadas; sin llegar a ser una antípoda de la otra conservan un peculiar entramado de relaciones. El insólito epistemólogo de la ciencia que fue Gastón Bachelard $(2004,2005)$ lo comprendió con claridad: la realidad cotidiana es el reino del sentido común, mientras que el territorio de la ciencia es un proyecto de construcción cognoscitiva y en cuanto tal se convierte en un poderoso ariete lanzado contra la ciudadela del sentido común. De hecho la ciencia consiste en un progresivo distanciamiento del mundo de la vida cotidiana y, agregaría Bachelard (2004, 2005): el conocimiento científico avanza por progresivas fases de abstracción. Por lo que la ciencia es más ciencia en la medida que se encuentra mayormente distante del mundo incierto del sentido común. Ahora bien, esto no implica que la ciencia acabe convirtiéndose en una nebulosa de abstracción sin ningún contacto con la realidad. Mientras más racionalmente es elaborado el conocimiento científico establece un más sofisticado entramado de relaciones con la realidad inmediata, lo que permite un conocimiento de aspectos inéditos o no concebidos previamente de la realidad; un ejemplo es la física cuántica que desafía hasta sus cimientos el sentido común. Aunque pareciera que este modelo de ciencia sólo responde al de las ciencias naturales, es de añadir que en cuanto a las ciencias humanas y sociales no es tan diferente, sobre todo si lo consideramos no desde el punto de vista de los objetos de ambos grupos de ciencias. Por supuesto que los objetos de las ciencias naturales y exactas no es el mismo que el de las ciencias humanas y sociales, pero si nos ubicamos en el terreno procedimental epistemológico ambos grupos de ciencias pueden seguir sendas análogas y comulgar en el mismo cáliz que ofrece Bachelard (2004, 2005). Por mencionar un ejemplo de esto en el caso de las ciencias sociales es la sociología de Pierre Bourdieu.

Si continuamos con la cuestión epistemológica, al ubicarnos ahora en el ámbito de la ciencia y su camino hacia la elaboración abstracta, debemos señalar que ese camino se encuentra asfaltado por los conceptos y la solidez y direccionalidad de la teoría. De manera simplificada podemos decir que una teoría es un sistema estructurado lógicamente por conceptos. Sin entrar en la litigiosa clasificación de conceptos de la lógica de raigambre aristotélica, sostenemos que los conceptos dentro de una arquitectura teórica tienen distintas disposiciones y funciones que les permiten llevar a cabo la construcción teórica de los objetos y prácticas de forma cada vez más abstracta en las diferentes ciencias particulares. Los objetos construidos teóricamente por mediación de los conceptos o, mejor aún, por un sistema relacional de conceptos se constituyen en el basamento sobre el que se levanta la fundamentación científica de una ciencia sea natural o social. Esta argumentación conceptual funge como marco para ubicar las nociones de tiempo y memoria, lo que implica que ahora son comprendidas desde supuestos epistemológicos, por lo que se estatuyen como conceptos explicativos de procesos cognoscitivos dentro de las ciencias de la información y que a su vez se correlacionan de diversa manera con los ya explicados fenómenos de tiempo y memoria informacionales del mundo de la vida cotidiana.

Una vez más hay que puntualizar que son diferentes el símbolo de tiempo y su correlativo de memoria como se expresan socialmente (explicitados por Elías) y los conceptos de tiempo y memoria como se instrumentan en la ciencia. Estos últimos son entidades construidas abstractamente por lo que sus disposiciones y funciones se manifiestan de manera específica por lo que pueden caracterizarse como conceptos soporte-operativos a diferencia de aquellos otros que son constructivo-explicativos. De hecho en el sistema interrelacional de conceptos aquellos de carácter soporte-operativos son el basamento sobre el que se levantan y desenvuelven los conceptos constructivo-explicativos. Tiempo y memoria en cuanto conceptos soporte-operativos tienen la disposición de ser soporte del concepto o los conceptos que se implementan para llevar a cabo la construcción abstracta (teórica) de los objetos y prácticas de conocimiento de la ciencia en particular en que se lleva a cabo tal proceso epistemológico; asimismo estos conceptos son operativos por qué acentúan al interior de los conceptos constructivo-explicativos: así tiempo y memoria se encuentran como la base en la que se apoyan los demás conceptos, pero no son un soporte estático sino que operan dentro de aquellos conceptos 
que al desplegar sus facultades constructivo-explicativas se encuentran signados por la temporalidad y la memoria; lo cual va modulando las facultades constructivo-explicativas de tales conceptos.

Veamos por medio de la ejemplificación esta explicación de orden conceptual; para ello empleamos como marco de referencia una de las ciencias de la información: la bibliotecología. Un tema que gradualmente ha adquirido una preponderancia estratégica dentro de esta ciencia es el de la lectura, lo que ha redundado en un incremento de investigaciones desde distintos enfoques sobre esta práctica, pero es de acotar que tales investigaciones en gran parte son de carácter superficial, es decir, resbalan por la superficie sin ir más allá. Sólo muy pocas de tales investigaciones buscan la fundamentación conceptual y teórica del tema de la práctica de lectura, la importancia de llevar a cabo la construcción conceptual y teórica de tal práctica, construyéndola así como objeto de conocimiento fundamentado bibliotecológicamente. Con ello se da un paso más para alcanzar el estatus de cientificidad de la bibliotecología. Tomemos, por tanto, la construcción como objeto de conocimiento la práctica de lectura; para lo cual los conceptos constructivo-explicativos de que dispondremos son los de intermediario, mediador y traducción, acuñados por el sociólogo Bruno Latour y que nos explica a continuación:

Un intermediario, en mi vocabulario, es lo que transporta significado o fuerza sin transformación: definir sus datos de entrada basta para definir sus datos de salida. Para todo propósito práctico un intermediario puede considerarse no sólo una caja negra sino también una caja negra que funciona como una unidad, aunque internamente esté compuesta de muchas partes. Los mediadores, en cambio, no pueden considerarse sólo uno; pueden funcionar como uno, nada, varios o infinito. Sus datos de entrada nunca predicen bien los de salida; su especificidad debe tomarse en cuenta cada vez. Los mediadores transforman, traducen, distorsionan y modifican el significado o los elementos que se supone que deben transportar. No importa lo complicado que sea un intermediario, puede representar, para todo propósito práctico, una unidad o incluso nada porque puede ser fácilmente olvidado. Por simple que pueda parecer un mediador, puede volverse complejo; puede llevar en múltiples direcciones que modificaran todas las descripciones contradictorias atribuidas a su rol (Latour, 2008, p. 63).

Como se desprende de sus palabras, el concepto de traducción va estrechamente unido al de mediador, y este y el intermediario se encuentran claramente diferenciados en cuanto lo que representan y significan. Ambos, y es preciso resaltarlo, son conceptos de orden relacional y en cuanto tal manifiestan dinámica, movimiento que transporta contenidos, con los que se correlacionan sujetos, acciones y objetos. La diferencia radica en que el intermediario transporta significado, pero no da lugar a transformación, por lo que es predecible en cuanto a los datos que vehiculiza. Por su parte, el mediador es heterogéneo en su atributo de vehiculizar múltiples significados; transfigura el significado de los elementos. Y esto se realiza a partir de la traducción: es el factor que propicia la transformación de aquello que es transportado. Intermediario y mediador-traducción son los conceptos constructivo-explicativos cuya cualidad epistemológica es que son de orden relacional, por lo que el objeto en proceso de construcción cognoscitiva que es la práctica de la lectura y el otro objeto elegido en este caso, de manera lógica, con el que entabla relación es el texto. Y los conceptos de soporte-operativos son los de tiempo y memoria.

Desde la perspectiva de la intermediación, la lectura al correlacionarse con el texto transporta un significado, que en cuanto tal es la información, pero el significado no se altera, esto es, que lo leído no produce cambios, queda igualmente como información en el lector; el cual, pasa a la lectura de otro texto y otro texto y otro... quedando solo como datos informativos sin transformación en él. Por el contrario, desde la explicación del mediador, la lectura de los textos al cruzar por la traducción cambia la información leída, se transforma en conocimiento, que pasa a ser una significación (información) diferente a la que lector tenía previo a su lectura. Lo que implica que la traducción se significa como una intervención distinta sobre el texto a la de mera descodificación informativa: extraña asumir la lectura de manera reflexiva y crítica, problematizándola.

Ahora bien, a la base de los conceptos de intermediario y mediador-traducción se encuentran los conceptos de tiempo y la memoria. Tanto el intermediario y el mediador-traducción se desenvuelven en una temporalidad. La lectura de los textos se lleva a cabo durante extensiones temporales, no es una actividad estática (en ninguno de sus gestos, sea lectura en silencio u oralizada, en una biblioteca o en una plaza pública, 
hundido en un sofá o caminando...); la temporalidad le otorga legibilidad de sentido a la práctica de lectura y a la asimilación de la información que ella brinda. Por su parte la memoria se manifiesta en dos vertientes: la memoria del lector y la memoria contenida en los textos. En la lectura como intermediario, la memoria del lector al contacto con la información del texto la reproduce como dato integrado a su propia memoria. Por su parte, la lectura en cuanto mediador implica que la memoria del lector al cruzar por la traducción transfigura la información textual (memoria contenido en el texto y que es producto, más allá de la particular individualidad del autor, del extenso afluente informativo del proceso civilizatorio), lo cual a su vez actúa recursivamente sobre la memoria (información) de lector propiciando la producción de conocimiento; con lo que se realiza de forma mayormente depurada la individualización del proceso civilizatorio. Es de añadir que los conceptos soporte-operativos por su disposición y función no se exteriorizan de manera explícita: actúan como un supuesto subrepticio y que únicamente se exteriorizan bajo ciertas condiciones y fases del proceso de conocimiento.

La construcción conceptual de los objetos y las prácticas no debe ser concebida únicamente como un oropel epistemológico de una ciencia, sino como un proceder cognoscitivo sistemático que ha de mostrar su capacidad explicativa de los objetos, prácticas y procesos de la realidad. Si continuamos con la línea discursiva previa, vemos cómo desde el ejemplo utilizado de la construcción conceptual de la práctica de lectura en bibliotecología se da explicación de los fenómenos correlativos de la realidad. Para lo cual nos remitimos a la exposición y reflexión que sobre el tiempo y la memoria nos ofreció Elías: la lectura es una práctica social que se desenvuelve básicamente como intermediario o mediador-traducción y ambas vías de lectura se despliegan en el tiempo a la manera en como explicó Elías lo hace este símbolo social. El largo proceso civilizatorio, el cual ha sido estudiado con lucidez a lo largo de toda su obra, muestra cómo las formas civilizatorias se han conformado como instancias sociales de coacción que al ser interiorizadas se individualizan en las conciencias de las personas ponen límites a sus pulsiones propiciando normas civilizadas de conducta y convivencia (Elías, 2016).

Sin embargo, de esa explicación se desprende el papel jugado por la transmisión de información que pasa de una generación a otra y que va constituyendo gradualmente las normas civilizatorias. En la transmisión de información jugó un rol fundamental esa memoria institucional que es la biblioteca, que al transmitir la información en ella preservada, el recuerdo como mecanismo social de recuperación, daba continuidad esa información. La vía para acceder a tal información ha sido y es la lectura, la cual se bifurca en cuanto intermediario que sólo reitera y da continuidad a la misma información recibida; y como mediador que cruza por la traducción dando lugar a conocimientos diferentes y nuevos. Una y otra formas de contacto con la información se incorporan al gran afluente civilizatorio, con lo que sigue su movimiento en el tiempo y memoria civilizatorio. Ambas prácticas de lectura y la respectiva expresión informativa a que dan lugar pasan por la individualización y es así preservada en la memoria de las personas y de ahí es comunicada entre ellas. Y si tales personas son lectoras esa información preservada en la memoria entra en contacto con la memoria contenida en el texto, como ya sé explicó. Según sea el tipo de lectura que hagan (intermediario o mediador-traducción), para comunicarse el símbolo social del tiempo, lo que les sirve para las percepciones de las relaciones entre los acontecimientos en que se encuentran inmersos los individuos. Con ello se constituyen el marco de referencia para orientarse socialmente. La información obtenida a través de ambos tipos de lectura les da, asimismo, el conocimiento para orientarse mejor. Es de acotar, y todo esto comprendido desde el escorzo de la práctica de lectura, esa orientación social que entraña la comunicación del símbolo de tiempo (y la memoria) en la medida que tiene como referente la lectura en cuanto mediador-traducción ofrece, valga la analogía, una brújula más efectiva para orientarse socialmente. 


\section{Corolario}

El tomar como ejes rectores de la argumentación desarrollada al tiempo y la memoria nos permitió comprender el rol fundamental que juegan ambas nociones dentro de las ciencias de la información. Y ello a partir de mostrar también su elaboración epistemológica, lo que dio pauta para exponer la necesidad de que en las susodichas ciencias se lleve a cabo un sistemático proceder de elaboración cognoscitiva de sus diversos objetos y prácticas específicas con lo cual sus procesos de conocimiento serán más consistentes y rigurosos.

Tiempo y memoria son, finalmente, el símbolo luminiscente que orienta socialmente a las ciencias de la información.

\section{REFERENCIAS}

Bachelard, G. (2004). La formación del espiritu cientifico. Contribución a un psicoanálisis del conocimiento objetivo. México: Siglo XXI.

Bachelard, G. (2005). El compromiso racionalista México: Siglo XXI.

Elías, N. (1989). Sobre el tiempo, Madrid: FCE.

Elías, N. (2016). El proceso de la civilización. Madrid: FCE.

Latour, B. (2008). Reensamblar lo social. Una introducción a la teoría del actor-red. Buenos Aires: Manantial. 